\title{
Phase determination of $x$-ray reflection coefficients
}

\author{
K.-M. Zimmermann, M. Tolan, R. Weber, J. Stettner, A. K. Doerr, and W. Press \\ Institut für Experimentelle und Angewandte Physik, Christian-Albrechts-Universität Kiel, Leibnizstraße 17-19, 24098 Kiel, Germany
}

(Received 4 May 2000; revised manuscript received 29 June 2000)

\begin{abstract}
It is shown to what extent the phase of a reflection coefficient may be determined from a single x-ray reflectivity experiment. Complex calculus guarantees that the so-called "phase problem" is relaxed for certain situations and the reflection coefficient is only determined by its moduls. A procedure to reconstruct the phase from a single measurement under incorporation of preknowledge of the system is derived and tested by numerical examples.
\end{abstract}

\section{INTRODUCTION}

In the last decades x-ray reflectivity has been successfully applied to investigate the electron density profiles of many thin film materials. ${ }^{1}$ However, one should always bear in mind that the density profiles obtained may not be unique, since they were generated in almost any case by fitting rather than by a direct data inversion. In general, the reconstruction of a density profile from a single reflectivity measurement is impossible. Phase information is irretrievably lost by observing intensities rather than field amplitudes. This inevitable fact is well known as the "phase problem" of x-ray scattering.

X-ray reflectivity may be discussed in terms of a purely optical language. The reflectivity of a single surface is given by the well-known Fresnel formulas. ${ }^{2}$ The only difference to conventional optics is that the real part of the refractive index $n$ for $\mathrm{x}$ rays is slightly smaller than unity, i.e., $n=1$ $-\delta+i \beta$, where the dispersion $\delta$ is positive and proportional to the electron density $\varrho$ of the material and $\beta$ accounts for absorption. The reflectivity for arbitrary electron density profiles $\varrho(z)$ may be calculated by slicing such a profile into very thin slabs of constant density and calculating the reflected intensity from these slabs via the famous Parratt-iteration $^{3}$ or the matrix method introduced earlier by Abelès. 4

A different description which is more common in the $\mathrm{x}$-ray scattering community is the kinematical or first-order Born approximation. The kinematical approximation allows a clearer treatment of the scattering, from which general conclusions may be more easily drawn. In the kinematical or "weak scattering" limit the cross section is proportional to the Fourier transform of the total three-dimensional electron density $\varrho(x, y, z)$ of the scatterer. For surfaces this can be reformulated, and the following expression results: $:^{5,6}$

$$
\begin{aligned}
R\left(q_{z}\right) & =R_{\mathrm{F}}\left(q_{z}\right)\left|\frac{1}{\varrho_{\infty}} \int \frac{d \varrho(z)}{d z} \exp \left(-i q_{z} z\right) d z\right|^{2} \\
& =R_{\mathrm{F}}\left(q_{z}\right)\left|F\left(q_{z}\right)\right|^{2}
\end{aligned}
$$

for the reflectivity $R\left(q_{z}\right)$. Here $q_{z}$ denotes the vertical wavevector transfer, $\varrho(z)$ is the laterally averaged electron density profile, and $\varrho_{\infty}$ is the average density of the entire sample. The prefactor $R_{\mathrm{F}}\left(q_{z}\right)$ is the usual Fresnel reflectivity of the substrate. ${ }^{6}$ It is worth noting that the structure factor $F\left(q_{z}\right)$ is associated with the derivative of the density profile $d \varrho(z) / d z$ by a simple one-dimensional (1D) Fourier transform.

Equation (1) has been discussed in great detail in the past. For a rough interface located at $z=0$ with an error-function profile of width $\sigma$ one gets $d \varrho(z) / d z \sim \exp \left\{-z^{2} /\left(2 \sigma^{2}\right)\right\}$, and thus $F\left(q_{z}\right)=\exp \left(-q_{z}^{2} \sigma^{2} / 2\right)$ and $R\left(q_{z}\right)=R_{\mathrm{F}}\left(q_{z}\right) \exp \left(-q_{z}^{2} \sigma^{2}\right){ }^{7}$ Various authors have shown that a direct consequence of the phase problem is that one can hardly expect to obtain more than that $\sigma$ value, the rms roughness, from a reflectivity measurement of a single surface. ${ }^{8-11}$ Thus, deviations from an error-function profile are hard to observe by $\mathrm{x}$-ray reflectivity in the special case of a single interface, such as for instance the surface of a bulk liquid. Even worse, it can be proven rigorously that asymmetries of such a profile cancel totally by taking the modulus squared in Eq. (1). ${ }^{10}$

However, the situation is not as bad as it is described in many works. For instance, by introducing a simple phase factor into the expression for $F\left(q_{z}\right)$ in Eq. (1) it is often claimed that the trivial cancellation of this factor by taking the modulus serves arbitrarily as a source for many ambiguities in the interpretation of $x$-ray reflectivty data. ${ }^{8}$ The aim of this paper is to show that in general this opinion is not true: It will be shown that the above-mentioned situation for a single surface improves considerably if thin film systems are considered. Then the reflection of $\mathrm{x}$ rays from interfaces at different depths provides the necessary phase information which may be used for the data analysis.

It has been shown that reflectivity data can be directly inverted if more than one measurement can be done on the same sample. For $\mathrm{x}$ rays this is possible whenever an absorption edge of the substrate is available. From the reflectivities at and away from this edge the density profile of the sample may be unambiguously retrieved. ${ }^{12,13}$ Other schemes propose the introduction of reference layers or they deal with the case of neutron reflectivity where spin polarization allows more than one measurement. ${ }^{14-19}$ However, these methods are subject to many practical limitations since often there is no appropriate adsorption edge. It is also not possible to introduce a reference layer without disturbing the original system and this reference layer introduces new ambiguities.

Furthermore real space approaches have been proposed where the Fourier transform of the reflection coefficient is discussed. Then a density-density correlation function (1D 
Patterson function) is obtained which may be directly related to a vertical length scale of the system under consideration..$^{20,21}$ Recently, the sensitivity of this approach has been improved considerably by special truncation techniques of the Fourier transform. ${ }^{22}$ It turns out that particularly for low contrast systems the transformation to real space may enhance features in a reflectivity which would otherwise remain invisible. In the present work the way we propose to find small features of a density profile from a single measurement is totally different from these approaches: A direct way of data inversion by a phase approximation is proposed. Recently the experimental feasibility of such phase approximations in the context of the full dynamical scattering theory has been discussed by van der Lee. ${ }^{23}$ However, we follow the original proposal of Sanyal and co-workers ${ }^{24,25}$ who applied phase approximations to special systems. In the present work we will show a general treatment which is based on the simple kinematical approximation. The kinematical approximation is easy to implement and has the advantage that results may be discussed analytically in a wider context. We found that our inversion scheme is valid for a large variety of systems.

The paper is structured as follows: First the outline of the theory is given. A formula is derived within the kinematic approximation which is well suited for a phase approximation. In the next paragraph it will be shown how these considerations may be converted into an algorithm to invert $\mathrm{x}$-ray reflectivity data. Here the inclusion of preknowledge about the system is of decisive importance. The next section deals with numerical examples in order to test the presented theory. It will be shown to what extent the developed method is able to yield reliable results. Conclusions and an outlook are given at the end of this paper.

\section{THEORY}

We consider the structure factor $F\left(q_{z}\right)$ as defined by Eq. (1), i.e.,

$$
F\left(q_{z}\right)=\frac{1}{\varrho_{\infty}} \int \frac{d \varrho(z)}{d z} \exp \left(-i q_{z} z\right) d z .
$$

This function may be analytically continued to the entire complex plane and may be expressed by its (known) modulus $\left|F\left(q_{z}\right)\right|$ and (unknown) phase $\Phi\left(q_{z}\right)$ via $F\left(q_{z}\right)$ $=\left|F\left(q_{z}\right)\right| \exp \left\{i \Phi\left(q_{z}\right)\right\}$. In the kinematic limit $q_{z}$ strictly is a real variable. ${ }^{26}$ For mathematical convenience $q_{z}$ is now treated as a complex variable. Then $F\left(q_{z}\right)$ is an analytic function whose properties may be discussed by complex calculus. It turns out that if the complex numbers $\left\{a_{1}, a_{2}, \ldots, a_{n}\right\}$ are a set of zeros of $F\left(q_{z}\right)$ all lying in the upper half plane, i.e., $\operatorname{Im}\left(a_{j}\right)>0$ for all $j=1, \ldots, n$, then the function $F\left(q_{z}\right)$ may be expressed by the following product: $^{27-29}$

$$
F\left(q_{z}\right)=\left|F\left(q_{z}\right)\right| \exp \left\{i \Phi_{H}\left(q_{z}\right)\right\} \prod_{j=1}^{n}\left(\frac{q_{z}-a_{j}}{q_{z}+a_{j}} \frac{q_{z}+a_{j}^{*}}{q_{z}-a_{j}^{*}}\right),
$$

where the asterisk marks a complex conjugate quantity and the so-called Hilbert phase $\Phi_{H}\left(q_{z}\right)$ is given by

$$
\Phi_{H}\left(q_{z}\right)=-\pi+\frac{2 q_{z}}{\pi} \int_{0}^{\infty} \frac{\ln \left[\left|F\left(q_{z}^{\prime}\right)\right| /\left|F\left(q_{z}\right)\right|\right]}{q_{z}^{\prime 2}-q_{z}^{2}} d q_{z}^{\prime} .
$$

Note the fact that the Hilbert phase depends on the moduls of $F\left(q_{z}\right)$ alone. A simple way to perform the numerical calculation of the Hilbert phase $\Phi_{H}\left(q_{z}\right)$ avoiding the singularity in the denominator of the integral in Eq. (4) is given in Ref. 29. From Eqs. (3) and (4) the phase of $F\left(q_{z}\right)$ may be expressed by its modulus and the zeros in the upper half complex plane by

$$
\Phi\left(q_{z}\right)=\Phi_{H}\left(q_{z}\right)+\sum_{j=1}^{n} 2 \arccos \left(\frac{2 q_{z} \operatorname{Im}\left(a_{j}\right)}{q_{z}^{2}-\left|a_{j}\right|^{2}}\right) .
$$

This important result transforms the phase problem to the (unknown) zeros of the analytic function $F\left(q_{z}\right)$ in the upper half plane. In particular, if no such zeros exist then $F\left(q_{z}\right)$ and hence the X-ray reflectivity given by Eq. (1) is fully determined by $\left|F\left(q_{z}\right)\right|$, from which the phase $\Phi\left(q_{z}\right)$ may be calculated via Eq. (4) since it coincides with the Hilbert phase. This is the content of the well-known Titchmarch theorem of complex calculus. Equation (5) directly visualizes that only the second part of the expression on the righthand side is unknown and hence the phase $\Phi\left(q_{z}\right)$ is indeed not completely random. Even if $F\left(q_{z}\right)$ would have zeros in the upper half complex plane then the sum which is running over all such zeros may nevertheless be small compared with the Hilbert phase $\Phi_{H}\left(q_{z}\right)$ and Eq. (5) would still be a good approximation of the unknown phase $\Phi\left(q_{z}\right)$.

For profiles fulfilling the condition $F\left(q_{z}\right) \neq 0$ in the upper half complex plane the phase problem does not exist. An extensive discussion of such profiles has been presented in the pioneering work of Clinton ${ }^{30}$ (see also Ref. 31 ). He has proven that a special class of functions obeys this requirement, namely, a layer stack with sharp interfaces and constant electron densities where the contrast between the substrate and the first layer is larger than the sum of all other contrasts. Thus, there exist - at least in theory-profiles $\varrho(z)$ for which a single reflectivity measurement already consists of all the necessary information to recover the phase of the reflection coefficient. The situation is somewhat more complicated if roughness is taken into account. But still there exists a large variety of density profiles where the phase of the reflection coefficient is totally determined by its modulus. ${ }^{30,31}$ Thus, the general statement that phase information is always completely lost and arbitrary phase factors may be introduced in Eq. (1) is not true.

It shall be further noted that all arguments given above are also valid if the exact dynamical treatment of x-ray reflectivity would be performed. However, since this complicates the calculations considerably we will not go into more details here. ${ }^{23,27,32-34}$

\section{INVERSION SCHEMES}

The considerations of the previous paragraph shall now be used to develop an algorithm for the inversion of x-ray reflectivity data. For this purpose we need a start profile $\varrho_{0}(z)$ 

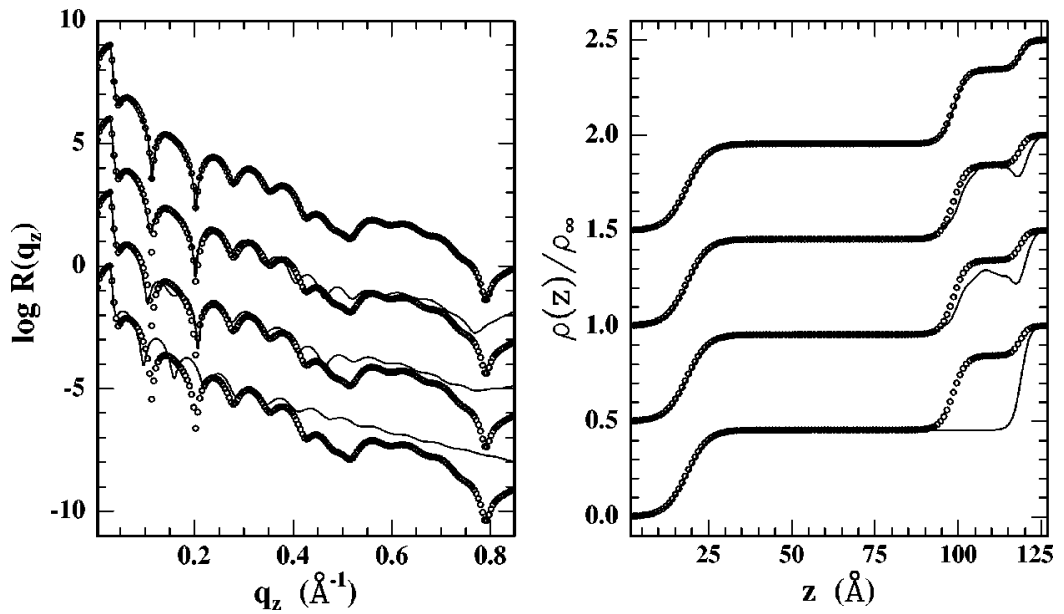

FIG. 1. Right panel: Unknown profile (open circles), first guess (lowest solid line) and the results after one iteration (second solid line from the bottom), three iterations (third solid line from the bottom), and more than 100 iterations (topmost curve) of the inversion algorithm described in the text. Left panel: Calculated reflectivities $R\left(q_{z}\right)$ corresponding to the density profiles in the right panel (open circles correspond to the unknown profile, solids lines to the respective iterated profiles in the right panel). All curves are shifted vertically for clarity.

which contains the preinformation that is known about the system (estimated layer thicknesses, mean layer densities, number of layers, etc.). Then the difference between this profile from the unknown profile $\varrho(z)$ is given by

$$
\Delta \varrho(z)=\varrho(z)-\varrho_{0}(z)
$$

Introducing this into Eq. (1) yields

$$
\left|F\left(q_{z}\right)\right|^{2}=\left|F_{0}\left(q_{z}\right)+\frac{i q_{z}}{\varrho_{\infty}} \Delta \tilde{\varrho}\left(q_{z}\right)\right|^{2},
$$

where $F_{0}\left(q_{z}\right)$ is the (known) 1D structure factor corresponding to $\varrho_{0}(z)$ as defined by Eq. (2) and $\Delta \widetilde{\varrho}\left(q_{z}\right)$ is the (unknown) Fourier transform of $\Delta \varrho(z)$. Equation (7) is solved formally by the ansatz

$$
\Delta \tilde{\varrho}\left(q_{z}\right)=\frac{i \varrho_{\infty}}{q_{z}}\left[F_{0}\left(q_{z}\right)-F\left(q_{z}\right)\right] .
$$

It is worth noting that Eq. (8) cannot be used to calculate $\Delta \widetilde{\varrho}\left(q_{z}\right)$ because only the modulus of $F\left(q_{z}\right)$ is known from a reflectivity measurement. Thus, $F\left(q_{z}\right)$ and $F_{0}\left(q_{z}\right)$ may be expressed in terms of the respective moduli and phases $\Phi\left(q_{z}\right)$ and $\Phi_{0}\left(q_{z}\right)$. Then Eq. (8) transforms to

$$
\begin{aligned}
\Delta \tilde{\varrho}\left(q_{z}\right)= & \frac{i \varrho_{\infty}}{q_{z}}\left[\left|F_{0}\left(q_{z}\right)\right|-\sqrt{\frac{R\left(q_{z}\right)}{R_{F}\left(q_{z}\right)}} \exp \left\{i \Delta \Phi\left(q_{z}\right)\right\}\right] \\
& \times \exp \left\{i \Phi_{0}\left(q_{z}\right)\right\},
\end{aligned}
$$

with the phase difference $\Delta \Phi\left(q_{z}\right)=\Phi\left(q_{z}\right)-\Phi_{0}\left(q_{z}\right)$ which is still unknown. However, Eq. (9) is well suited for a phase approximation based on the considerations of the previous paragraph. The simplest approximation of the phase difference is given by setting $\Delta \Phi\left(q_{z}\right) \equiv 0$. This means that the phases of the start profile and of the unknown profile already coincide very well, i.e., the second term on the right-hand side of Eq. (5) is small. Sanyal et al. ${ }^{24,25,36}$ have tested other inversion schemes based on the distorted wave Born approximation. For certain systems such as polymer films on silicon substrates or GaAs/AlAs multilayers they found good results with the approximation $\Delta \Phi\left(q_{z}\right) \equiv 0$.

A better way to use Eq. (9) for the inversion of x-ray reflectivity data is based on two improvements: (i) The phase difference $\Delta \Phi\left(q_{z}\right)$ may be approximated more accurately by the Hilbert phases of $F\left(q_{z}\right)$ and $F_{0}\left(q_{z}\right)$ as discussed in the previous paragraph [see Eq. (4)]. (ii) Equation (9) may be solved in an iterative way, i.e., the result for $\Delta \varrho(z)$ which is given by a Fourier backtransformation of the right-hand side of Eq. (9) may be used as the new known starting profile $\varrho_{0}(z)$. We introduced number (ii) into an algorithm which is otherwise based on Eq. (9). ${ }^{37}$ It turned out by numerical simulations that number (i), the introduction of the Hilbert phase difference instead of $\Delta \Phi \equiv 0$, only accelerates the convergence of the algorithm but this does not affect the final result. ${ }^{35}$ The possibility to impose additional restrictions on the expected profiles was also included. In particular, it turned out that often only a part of the profile, for instance a single interface structure, is unknown while the rest is completely determined by the preknowledge about the system. Then these known parts of the function $\varrho(z)$ were fixed and the algorithm was only applied for the rest of the profile. It has been proved mathematically that if certain areas of a profile are fixed then an unambiguous reconstruction of the remaining part is possible from a single reflectivity measurement. $^{27,28}$ However, since measurements always contain statistical errors and because they are limited to a finite $q_{z}$ range, in reality this may not be the case.

\section{NUMERICAL EXAMPLES}

We will now discuss numerical examples which show that the presented method works for a large variety of systems. All systems have in common that the reflectivities and electron density profiles for layer systems with silicon as substrate material are calculated. As a first guess a structureless polymer or otherwise organic film is always taken. The assumed optical parameters $\delta$ and $\beta$ of silicon and of the organic film correspond to those for radiation with a wavelength of $\lambda=1.54 \AA$ ( $\mathrm{Cu} K \alpha$ radiation). Furthermore, the reflectivities calculated with the exact Parratt formalism ${ }^{1,3,31}$ will be displayed together with the respective density profiles. Geometric factors as well as a standard resolution folding also have been included into the reflectivity calculations. $1,31,38,39$

Figure 1 shows a simple example. The right panel contains the density profiles. The open circles represent the "unknown" profile which is going to be reconstructed. The lowest solid curve is the first guess, i.e., the starting profile 

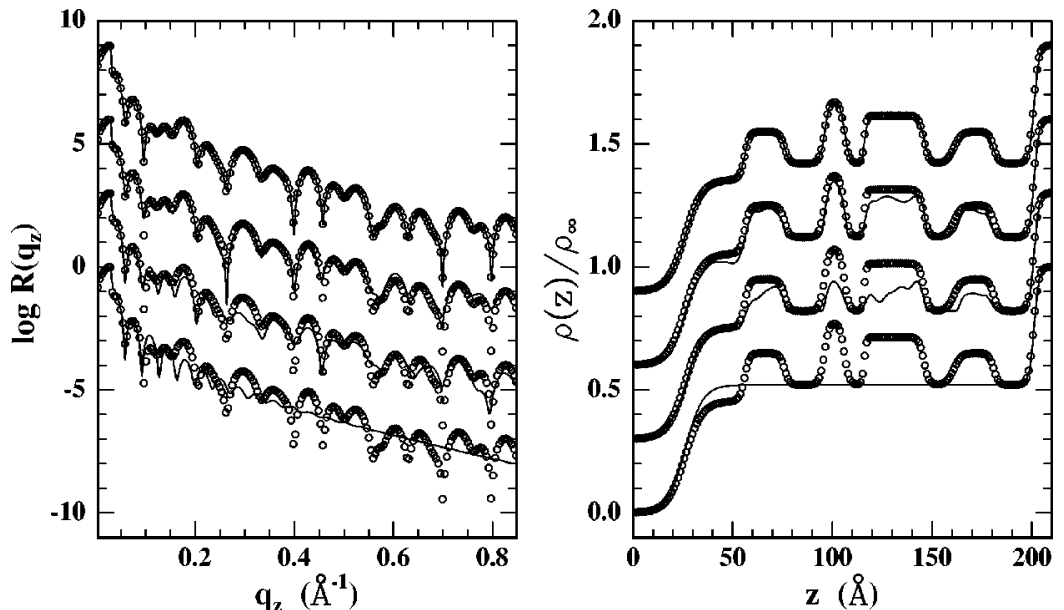

FIG. 2. Inversion of a more complicated layer structure (see open circles in the right panel). It shall be noted that the layers are not uniform. All other explanations are the same as for Fig. 1. $\varrho_{0}(z)$ of the iteration. It can be seen that obviously the layer next to the substrate is initially missing. The complete layer including the interface roughnesses will now be reconstructed. The left panel contains the corresponding reflectivities. The open circles show the reflectivity for the unknown profile. This reflectivity would be the result of a measurement from which $\left|F\left(q_{z}\right)\right|$ may be obtained according to Eq. (1). The lowest solid line is the reflectivity corresponding to the profile given by the first guess as discussed before. The two curves disagree considerably for large $q_{z}$ values. The second solid line from the bottom depicts the reflectivity as calculated for the second profile marked by the solid line in the right panel. This profile is obtained after the first iteration of the reconstruction algorithm presented in the last paragraph. It can be seen that the unknown layer already becomes visible. However, the reconstruction is not yet perfect. After three iterations the solid lines in the left and right panels (third curves from the bottom) indicate that the profile and the reflectivity converge towards the final solution. In the topmost curves the solid lines and the open circles coincide rather perfectly. These curves are obtained after more than 100 iterations. Thus, in this specific example it is demonstrated that a perfect reconstruction of an unknown layer from a single "measurement" is possible.

Figure 2 shows the next example with much more complexity. The description of the curves is similar to that given for Fig. 1. It can be seen that even quite complex unknown layer structures may be reconstructed from a single reflectivity curve and a minimum preinformation, namely, in this case only one layer with a mean density on a silicon substrate was assumed. ${ }^{40}$ However, in order to accelerate the covergence of the algorithm a lower limit for the electron density of the film was set. Such a limit is not a major restriction since the mean densities often are given by literature values.

These examples have demonstrated that the phase of an $\mathrm{x}$-ray reflection coefficient and hence the electron density profile of a layer system can indeed be reconstructed from a single reflectivity curve, i.e., from the measurement of the $x$-ray reflectivity alone. Even in cases where the calculated reflectivity for the first guess $\varrho_{0}(z)$ and the unknown profile $\varrho(z)$ are quite different on the entire $q_{z}$ range the proposed inversion scheme is able to generate the required solution (see Fig. 2). However, it is worth noting that this does not mean that the phase problem of x-ray scattering is solved. The reason why the inversion algorithm works is the input of the preinformation and for more complex systems the use of the Hilbert phase as discussed earlier. ${ }^{30}$ These two ingredients already pin down the phase for many systems.

If the structure factor $F\left(q_{z}\right)$ has zeros in the upper half plane and if the preknowledge is not sufficient for an unambiguous phase reconstruction then the result of the inversion algorithm may depend on the starting profile $\varrho_{0}(z)$. This is
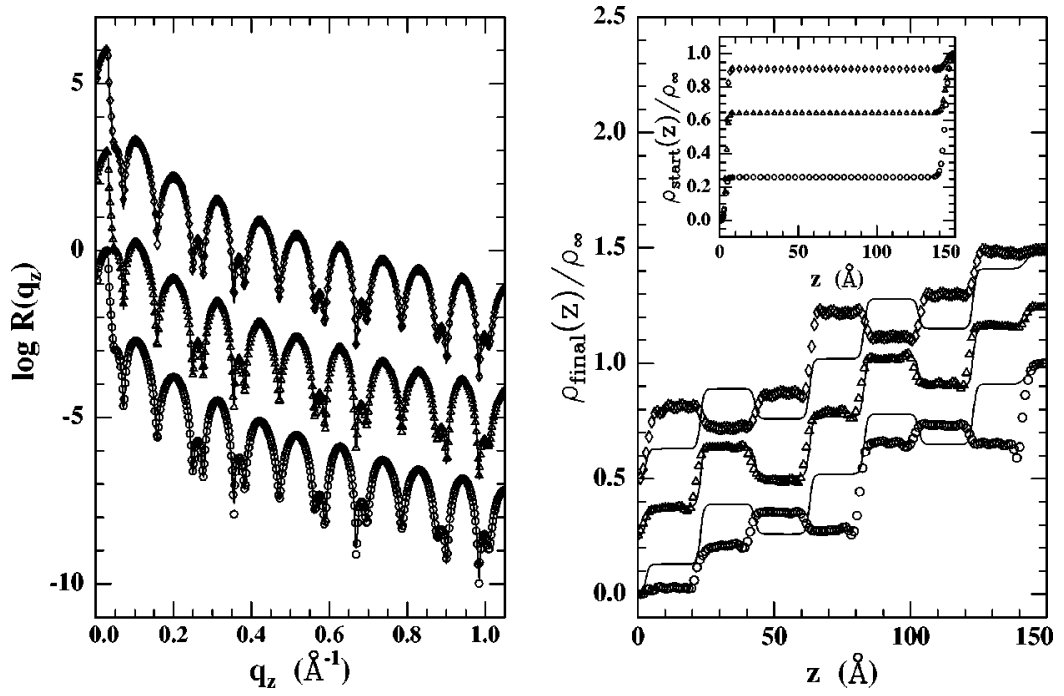

FIG. 3. Example where the inversion algorithm does not yield a unique solution. Right panel: The solid lines represent the unknown profile. The symbols are the results after more than 100 iterations of the algorithm described in the text using the start profiles depicted in the inset [the same symbols mark the start and final profiles, $\varrho(z)_{\text {start }}$ and $\varrho(z)_{\text {final }}$. Only the start profile in the middle (open triangles) yields the desired solution. The other start profiles converge to similar but wrong results. Left panel: Calculated reflectivities for the unknown profile (solid lines) and the results of the inversions (symbols) with the three start profiles shown in the inset of the right panel. All reflectivities agree. The density profiles and the reflectivities are vertically shifted for clarity. 


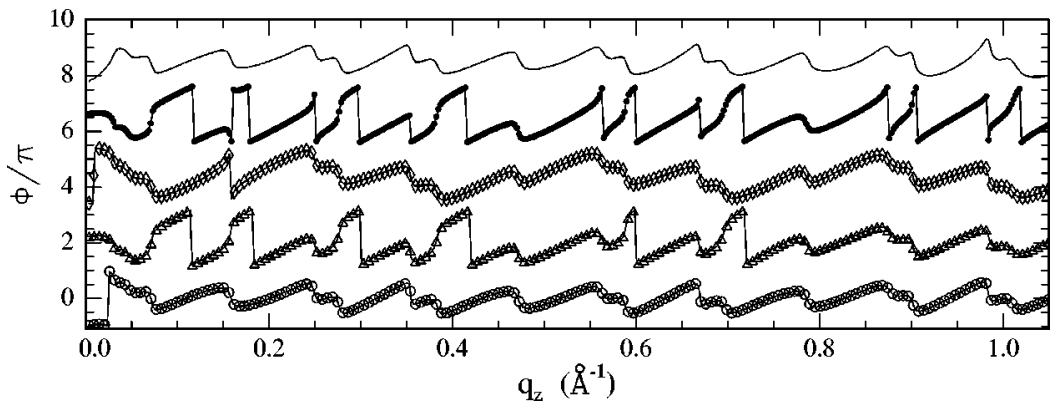

FIG. 4. Phases $\Phi\left(q_{z}\right)$ of the reflection coefficients corresponding to the density profiles shown in the right panel of Fig. 3. The topmost curve (solid line) is the Hilbert phase calculated for the unknown density profile. The second curve from the top (filled circles) is the exact phase for the unknown profile. Each of the other three curves corresponds to the reflection coefficient of the profile with the same symbol in the right panel of Fig. 3.

discussed in the next example which is presented in Fig. 3. This figure is differently organized compared to those before. The solid lines in the right panel display the unknown density profile $\varrho(z)$. An arrangement of equally spaced steps with similar interfaces is depicted. The inset shows three different starting profiles $\varrho_{0}(z)$ all consisting of one structureless layer on a substrate but with three different values of the mean density. A perfect reconstruction of the unknown profile is achieved only if the start profile in the middle (open triangles) is used. This is demonstrated by the open triangles in the right panel which represent the result of the inversion algorithm as discussed before. However, if the algorithm is started with the other two profiles then the upper and lower curves (open diamonds and open circles) in the right panel of Fig. 3 are obtained. Although they do contain characteristic features of the unknown profile it is obvious that they do not represent the desired solution. This is explained in the left panel of Fig. 3 where the calculated reflectivities are displayed. The solid lines show the reflectivity of the unknown profile, the symbols correspond to the respective profiles in the right panel. It can be seen that all calculated reflectivities coincide, i.e., for this special case the three density profiles cannot be distinguished.

The corresponding phases $\Phi\left(q_{z}\right)$ of the reflection coefficients are shown in Fig. 4. The solid line (upper curve) is the Hilbert phase of the reflection coefficient for the profile given by the solid line in the right panel of Fig. 3, the exact phase is given by the filled circles (second curve from the top). The two curves are quite different. The other three phases correspond to the reflection coefficients calculated for the three profiles in the right panel of Fig. 3 which were obtained after the inversion with the different start profiles $\varrho_{0}(z)$ as discussed above. It can be seen that the phase for the result given by the open triangles in Fig. 3 almost coincides with the exact phase of the system. Only the points where the exact phase is discontinuous were not well reproduced. This is an artifact caused by the truncation in order to perform the numerical Fourier transforms within the inversion algorithm. The other two phases (open diamonds and open circles) are similar to the Hilbert phase. This is the reason why these start profiles (see inset in Fig. 3) converge to similar but false results. Hence this example demonstrates that the phase problem - of course-has not been solved.

The considerations have shown that phase information from preknowledge of a system can be used to improve the analysis of $\mathrm{x}$-ray reflectivity data. In the previous examples the algorithm was only tested with smooth calculated curves. In order to test the inversion scheme in a more realistic situation we included noise into our calculated reflectivities. These noisy_but still artificial_ "data" allow a systematic investigation of the effect of the counting statistics on the inverted density profiles. ${ }^{23,34}$ It turns out that noisy data cause the largest uncertainties at the locations of the interfaces where $d \varrho(z) / d z$ changes rapidly over small vertical distances. This might be due to the numerical Fourier transforms. These transforms may become unstable for the profiles because the inversion algorithm searches for a perfect solution for each point of noisy high $q_{z}$ data. However, we found that the relative error $\delta \varrho(z) / \varrho(z)$ caused by the statistics is always less than $3 \%$ provided that the covered $q_{z}$ range is large enough and remarkable noise starts to disturb the data only for $q_{z}>0.4 \AA^{-1}$.

\section{CONCLUSIONS AND OUTLOOK}

We have presented calculations which reveal that the phase of a reflection coefficient may be reconstructed from a single $\mathrm{x}$-ray reflectivity measurement. Our inversion algorithm has been tested with systems of different degrees of complexity. It turns out that the inclusion of trivial preknowledge already approximates the phase of the reflection coefficient very well. The examples suggest that this is valid for a large variety of systems with rather complex structures. Thus, the general statement that phase information is completely lost by an $\mathrm{x}$-ray reflectivity measurement which may often be found in publications dealing with $x$-ray reflectivity (see, e.g., Ref. 8) is not at all true. Such a statement is also in disagreement with Eq. (5), which shows that only a part of the phase is unknown.

Phase information is always present whenever interferences are observed stemming from $\mathrm{x}$ rays scattered from different depths of a sample. Thus, if the mean film thickness of a single layer could be varied without disturbing the interface structures one would be able to change the phase of the reflection coefficient in a controlled manner. This scenario experimentally is almost present for liquid thin films where the film thickness is controlled via the vapor pressure above the liquid. Then a simultaneous inversion of a large set of curves 
yields unambiguously the common unknown interface structures. $^{35,41}$

Possible applications of our algorithm are $\mathrm{X}$-ray data which extend over a wide $q_{z}$ range and where tiny features in a profile are going to be found. Such profiles are expected for instance in the case of polymer or liquid films next to solid substrates $^{21,24,35}$ or for metal/polymer interfaces.

\section{ACKNOWLEDGMENTS}

This work was supported by the Deutsche Forschungsgemeinschaft Projects Nos. Pr325/12-1 and Pr325/5-2 within the Schwerpunktprogramm "Benetzung und Strukturbildung an Grenzflchen.'" M.T. would like to thank Professor M.K. Sanyal for many fundamental discussions about phases of reflection coefficients.
${ }^{1} X$-Ray and Neutron Reflectivity, edited by J. Daillant and A. Gibaud, Lecture Notes in Physics Vol. 58 (Springer-Verlag, Berlin, 1999).

${ }^{2}$ M. Born and E. Wolf, Principles of Optics (Pergamon, Oxford, 1993).

${ }^{3}$ L.G. Parratt, Phys. Rev. 95, 359 (1954).

${ }^{4}$ F. Abelès, Ann. Phys. (Paris) 5, 596 (1950).

${ }^{5}$ J. Als-Nielsen, D. Jacquemain, K. Kjær, F. Leveiller, and M. Lahav, Phys. Rep. 246, 251 (1994).

${ }^{6}$ M. Deutsch and B.M. Ocko, Encyclopedia of Applied Physics (Wiley-VCH, New York, 1998), Vol. 23, p. 479.

${ }^{7}$ P. Beckmann and A. Spizzichino, The Scattering of Electromagnetic Waves From Rough Surfaces (Pergamon, New York, 1963).

${ }^{8}$ P.S. Pershan, Phys. Rev. E 50, 2369 (1994).

${ }^{9}$ F. Rieutord, A. Braslau, R. Simon, H.J. Lauter, and V. Pasyuk, Physica B 221, 538 (1996).

${ }^{10}$ W. Press, J.-P. Schlomka, M. Tolan, and B. Asmussen, J. Appl. Crystallogr. 30, 963 (1997).

${ }^{11}$ T. Salditt, Ph.D. thesis, Universität München, 1995.

${ }^{12}$ M.K. Sanyal, S.K. Sinha, A. Gibaud, K.G. Huang, B.L. Carvalho, M. Rafailovich, J. Sokolov, X. Zhao, and W. Zhao, Europhys. Lett. 21, 691 (1993)

${ }^{13}$ T. Ohkawa, Y. Yamaguchi, O. Sakata, M.K. Sanyal, A. Datta, S. Banerjee, and H. Hashizume, Physica B 221, 416 (1996).

${ }^{14}$ D.S. Sivia, W.A. Hamilton, G.S. Smith, T.P. Riecker, and R. Pynn, J. Appl. Phys. 70, 732 (1991).

${ }^{15}$ V.O. de Haan, A.A. van Well, S. Andenwalla, and G.P. Felcher, Phys. Rev. B 52, 10831 (1995).

${ }^{16}$ V.O. de Haan, A.A. van Well, P.E. Sacks, S. Andenwalla, and G.P. Felcher, Physica B 221, 524 (1996).

${ }^{17}$ C.F. Majkrzak and N.F. Berk, Physica B 221, 520 (1996).

${ }^{18}$ C.F. Majkrzak, N.F. Berk, J.A. Dura, S.K. Satija, A. Karim, J. Pedulla, and R.D. Deslattes, Physica B 248, 338 (1998).

${ }^{19}$ A. Schreyer, C.F. Majkrzak, N.F. Berk, H. Grüll, and C.C. Han, J. Phys. Chem. Solids 60, 1045 (1999).

${ }^{20}$ G. Vignaud, A. Gibaud, G. Grübel, S. Joly, D. Ausserré, J.F. Legrand, and Y. Gallot, Physica B 248, 250 (1998).

${ }^{21}$ C.-J. Yu, A.G. Richter, A. Datta, M.K. Durbin, and P. Dutta, Phys. Rev. Lett. 82, 2326 (1999)

${ }^{22}$ O.H. Seeck, I.D. Kaendler, M. Tolan, K. Shin, M.H. Rafailovich, J. Sokolov, and R. Kolb, Appl. Phys. Lett. 76, 2713 (2000).

${ }^{23}$ A. van der Lee, Eur. Phys. J. B 13, 755 (2000).
${ }^{24}$ M.K. Sanyal, J.K. Basu, A. Datta, and S. Banerjee, Europhys. Lett. 36, 265 (1996).

${ }^{25}$ M.K. Sanyal, S. Hazra, J.K. Basu, and A. Datta, Phys. Rev. B 58, R4258 (1998).

${ }^{26}$ Absorption and refraction effects may be included in a semiempirical way into the kinematic theory by introducing a complex wave number (Ref. 8).

${ }^{27}$ K. Chadan and P.C. Sabatier, Inverse Problems in Quantum Scattering Theory, 2nd ed., Text and Monographs in Physics (Springer, Berlin, 1989).

${ }^{28}$ M.V. Klibanov and P.E. Sacks, J. Comput. Phys. 112, 273 (1994).

${ }^{29}$ G. Reiss, Physica B 221, 533 (1996).

${ }^{30}$ W.L. Clinton, Phys. Rev. B 48, 1 (1993).

${ }^{31}$ M. Tolan, X-Ray Scattering from Soft-Matter Thin FilmsMaterials Science and Basic Research, Springer Tracts in Modern Physics Vol. 148 (Springer, Berlin, 1999).

${ }^{32}$ R. Lipperheide, G. Reiss, H. Fiedeldey, S.A. Sofianos, and H. Leeb, Phys. Rev. B 51, 11032 (1995).

${ }^{33}$ R. Lipperheide, G. Reiss, H. Leeb, and S.A. Sofianos, Physica B 221, 514 (1996).

${ }^{34}$ R. Lipperheide, J. Kasper, and H. Leeb, Physica B 248, 366 (1998).

${ }^{35}$ A.K. Doerr, Ph.D. thesis, Kiel University, 1999.

${ }^{36}$ S. Banerjee, M.K. Sanyal, A. Datta, S. Kanakaraju, and S. Mohan, Phys. Rev. B 54, 16377 (1996).

${ }^{37}$ The algorithm was implemented using the language $\mathrm{C}^{2+}$ on a standard linux PC with AMD K6 $450 \mathrm{MHz}$ processor. 1000 iterations took a time of approximately 5 min depending on the layer thicknesses and the required resolution. The obtained profile was smoothend after the iterations by a convolution with a Gaussian in order to eliminate truncation effects caused by the Fourier transforms.

${ }^{38}$ A. Gibaud, G. Vignaud, and S.K. Sinha, Acta Crystallogr., Sect. A: Found. Crystallogr. 49, 642 (1993).

${ }^{39}$ V. Holý, U. Pietsch, and T. Baumbach, X-Ray Scattering from Thin Films (High Resolution X-Ray Scattering from Crystalline Thin Films), Springer Tracts in Modern Physics Vol. 149 (Springer, Berlin, 1999).

${ }^{40}$ To accelerate the convergence also the thin oxide layer of the substrate was assumed to be known. The inversion would also work without this preinformation.

${ }^{41}$ A.K. Doerr, M. Tolan, J.-P. Schlomka, and W. Press (unpublished). 